\title{
YIELD STRENGTH OF THIN-FILM PARYLENE-C
}

\author{
Victor Chi-Yuan Shih, Theodore A. Harder and Yu-Chong Tai \\ Caltech Micromachining Laboratory \\ Electrical Engineering Department MC136-93, California Institute of Technology \\ Pasadena CA91125 USA \\ Telephone: (626)395-2227 Fax(626)584-9104 \\ E-mail:cyshih@caltech.edu
}

\begin{abstract}
For the first time, the yield strength of thin-film parylene-c is measured from membrane load-deflection experiments and surface profile analysis. To do so, the onset pressure which causes plastic deformation of the membrane is first experimentally measured. Then a new 2-step displacement model, together with the energy minimization technique[1], is developed to convert the onset pressure to the yield strength on the pre-stressed parylene membrane under a uniform pressure loading. The results depict a Yield Strength of $59 \mathrm{MPa}$ (or 0.012 of strain) for thin-film parylene-c in comparison to $55 \mathrm{MPa}$ reported by parylene vendor (measured from large samples)[2]. To double check with the result, the balloon model[3] is further used to compare with the stress value from our model at the center of parylene membranes and good agreements are obtained.
\end{abstract}

\section{INTRODUCTION}

Parylene is getting more and more popular for MEMS applications such as for valves, pumps, nozzles, flow sensors and even accelerometers[4-7]. Unlike the conventional silicon material, however, parylene has a significant plastic deformation even at room temperature if it is stressed over the yield point. It is therefore very important to measure the not-yet-known yield strength of thin-film parylene so that the operation limit of devices made of parylene can be predicted. For parylene membranes under uniform pressure loading, the total stress is the sum of the intrinsic stress and the loading stress, while intrinsic stress (caused mainly by thermal mismatch between the membrane and the substrate) usually dominates[8]. Although stress distribution models for zero-intrinsic-stress, large-deflection membrane under loadings are available[1], there is the lack of a simple analytical model to cover both the effects of large intrinsic stress and large deflection existent at the same time in membranes[9][10]. Here we propose to use the membrane load-deflection method[11] to determine the plastic deformation onset pressure of thin-film parylene-c and we present a simple analytic modeling of it.

\section{FABRICATION AND SYSTEM SETUP}

The membranes used in the load-deflection test are $2.8 \times 1.6 \mathrm{~mm}^{2}$ with a thickness of $2.8 \mu \mathrm{m}$. Fig. 1 illustrates the fabrication process of the load-deflection membrane.

(a)

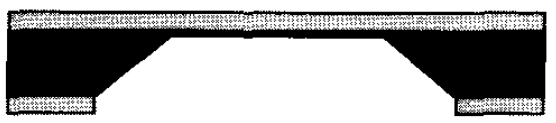

(b)

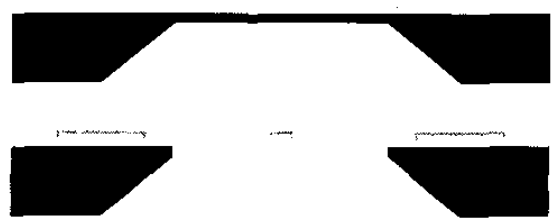

Fig.1 Fabrication process for load-deflection membrane. (a) Pattern oxide and $\mathrm{KOH} \mathrm{Si}$ etching to make Si membrane. (b) Strip oxide and deposit thin-film parylene c. (c) Deposit and pattern $\mathrm{Cr} / \mathrm{Au}$. $\mathrm{BrF}_{3} \mathrm{Si}$ etching to release parylene membrane.

First, a suitable silicon membrane is produced. The desired membrane pattern is etched into the oxide mask on the backside of the wafer. Then, $\mathrm{KOH}$ etching is used to produce silicon membranes with a thickness of around 20 $\mu \mathrm{m}$. Second, the remaining oxide is removed using BHF and then the wafer is placed in the parylene deposition chamber. A $2.8 \mu \mathrm{m}$ of parylene-c is deposited on the top side of the wafers. Third, $\mathrm{Cr} / \mathrm{Au}(10 \mathrm{~nm} / 200 \mathrm{~nm})$ is deposited on top of parylene with thermal evaporator and is patterned to generate a single $20 \times 20 \mu \mathrm{m}^{2} \mathrm{Cr} / \mathrm{Au}$ square 
in the membrane center to provide deflection reference points for later WYKO profile measurements. The wafer is then diced into individual chips at this point and annealed at $140{ }^{\circ} \mathrm{C}$ for one hour to ensure consistent intrinsic stress[11]. Finally, BrF3 slicon etching from the backside releases the parylene-c membrane from the silicon membrane.

The load-deflection measurements are made using a simple set of pressure regulators and needle valves for pressure regulation and a WYKO NT2000 interferometer for measuring the membrane deflection profiles. The setup is illustrated in Fig.2. The pressure regulator regulates between $0-30 \mathrm{psi}$ and the needle valves functioned as pressure dividers reducing the effective pressure into repeatable $0.01 \mathrm{psi}$ increments. The pressure was measured with an Omega PCL100-30 pressure calibrator with 0.001 psi resolution. The WYKO interferometer measures 3D surface profiles with an accuracy of $0.05 \mu \mathrm{m}$. However, inner reflection of WYKO light source from transparent parylene thin film introduces small profile offsets (a couple of microns) in the measurement. Therefore, small gold reference squares are fabricated in the center of each membrane and offmembrane regions to allow WYKO to record accurate maximum deflection of the membranes.

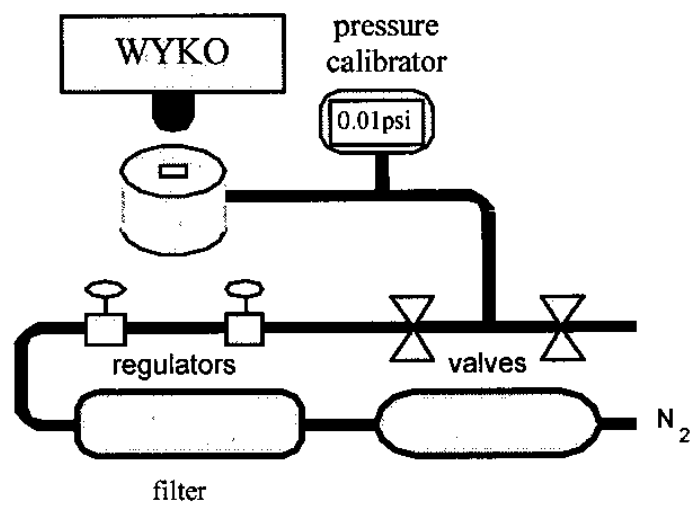

Fig.2 System setup for membrane load-deflection experiment.

\section{MEASUREMENTS AND ANALYSIS}

\section{A. Energy Minimization Method}

In order to measure the yield strength of pre-stressed thin-film parylene-c, the membranes are loaded into plastic deformation regime and we need to record the onset pressure that causes hysteresis in the load-unload deflection curves. This approach is different from the conventional brittle membrane experiment and is specially suitable for device performance characterization. Fig.3 shows an example of the hysteresis data from load-unload

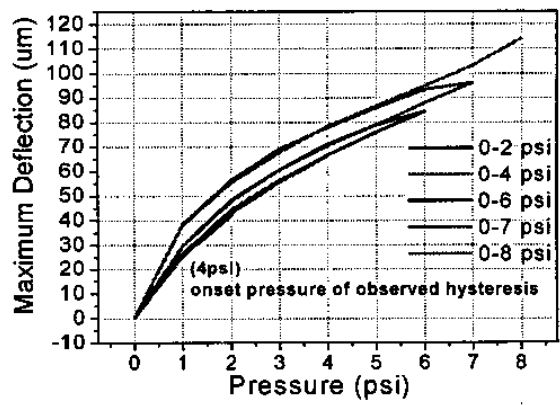

Fig.3 Load-deflection curves of parylene membranes under various pressure cycles. The maximum membrane deflection happens in the center of the membrane.

cycles with increasing pressures up to 8 psi. From data we observe 4 psi to be the lowest pressure showing hysteresis load-deflection curve. When large hysteresis happens $(\sim 8 \mathrm{psi})$, plastic deformation along the membrane long edges can be clearly seen in the WYKO profile (shown in Fig.4) which confirms the prediction from our model introduced later in this section. This way, we can accurately measure the onset hysteresis pressure and then, we use our new two-step displacement model with energy

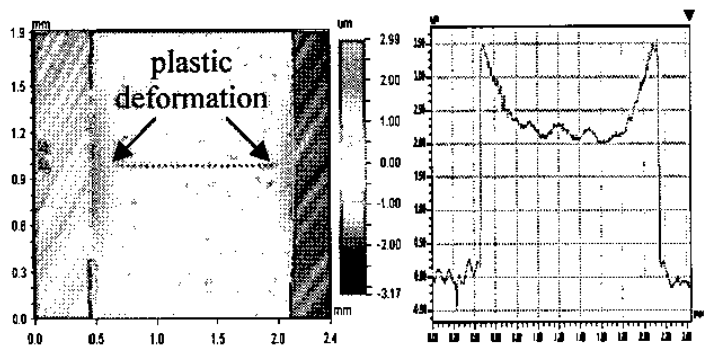

Fig.4 Plastic deformation observed on the long edge of parylene membrane after a 8psi loadunload cycle.

minimization method to interpret the data. The two-step displacement approach (Fig.5) assumes the membrane is first stretched from a stress-free status to the fabricated status which has a uniform tensile stress caused mainly by thermal mismatch between silicon substrate and parylenec membrane during the cooling process after the annealing. In the second step the membrane is further bulged with a uniform loading pressure. Displacements of 


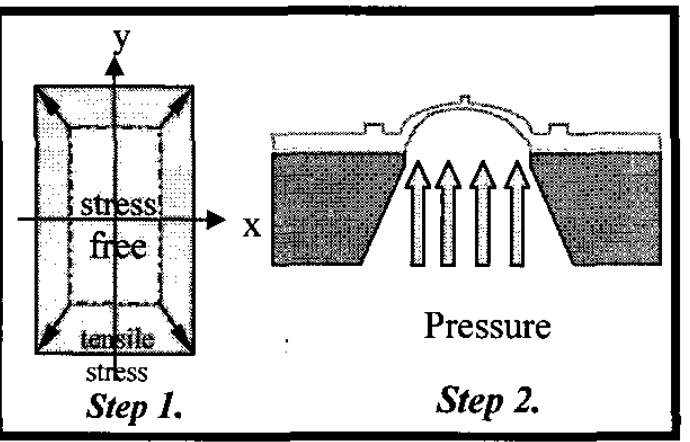

Fig.5 Two-step displacement model.

$u(x, y)=\varepsilon_{\text {in }} x+u_{0} \sin \frac{\left(1+\varepsilon_{\text {in }}\right) \pi x}{a} \cos \frac{\left(1+\varepsilon_{i n}\right) \pi y}{2 b}$

$v(x, y)=\varepsilon_{\text {in }} y+v_{0} \sin \frac{\left(1+\varepsilon_{\text {in }}\right) \pi y}{b} \cos \frac{\left(1+\varepsilon_{\text {in }}\right) \pi x}{2 a}$

$w(x, y)=w_{0} \cos \frac{\left(1+\varepsilon_{i n}\right) \pi x}{2 a} \cos \frac{\left(1+\varepsilon_{\text {in }}\right) \pi y}{2 b}$

$\varepsilon_{i n}=\frac{\sigma_{i n}}{E}(1-v) \quad \sigma_{\text {in }}:$ intrinsic stress, $v$ : poisson ratio

Eq.1 Displacement components derived from the two-step approach. $\mathrm{u}, \mathrm{v}$ and $\mathrm{w}$ are displacements in the $\mathrm{x}, \mathrm{y}$ and $\mathrm{z}$ directions respectively. $2 \mathrm{a}, 2 \mathrm{~b}$ are the width and length of the parylene membrane respectively. $u_{0}, v_{0}$ and $\mathrm{w}_{0}$ are parameters to be determined from energy minimization method.

any point on the membrane therefore has two parts (Eq.1) where $\mathrm{u}, \mathrm{v}$ and $\mathrm{w}$ are displacements in the $\mathrm{x}, \mathrm{y}$ and $\mathrm{z}$ directions respectively. The second part of the overall displacement in Eq.1 is an approximation from neglecting the higher order modes in an infinite summation expression first presented by Timoshenko[1][9]. More accurate analysis can be obtained if more high order modes are included in Eq.1. Energy minimization method is then used to analytically determine the displacement components across the whole membrane and the membrane load-deflection relationship as follows :

$u, v, w$ : displacements of points on the membrane in $x, y$ and $\mathrm{z}$ directions

$\varepsilon_{\mathrm{x}}, \varepsilon_{\mathrm{y}}$ : strain in the $\mathrm{x}$ and $\mathrm{y}$ direction

$V_{1}$ : energy due to stretching of the membrane

$\mathrm{V}_{2}$ : work done by the pressure to the membrane

p: applied pressure difference to the membrane $f, f_{1}, f_{2}, f_{3}$ : functions derived from the respective partial differential equation

$$
\begin{aligned}
& \varepsilon_{x}=\frac{\partial u}{\partial x}+\frac{1}{2}\left(\frac{\partial w}{\partial x}\right)^{2} \\
& \varepsilon_{y}=\frac{\partial v}{\partial y}+\frac{1}{2}\left(\frac{\partial w}{\partial y}\right)^{2} \\
& \gamma_{x y}=\frac{\partial u}{\partial y}+\frac{\partial v}{\partial x}+\frac{\partial w}{\partial x} \frac{\partial w}{\partial y} \\
& V_{1}=\frac{E \cdot t}{2\left(1-v^{2}\right)} \int_{-b-a}^{b} \int_{-a}^{a}\left[\varepsilon_{x}^{2}+\varepsilon_{y}^{2}+2 v \varepsilon_{x} \varepsilon_{y}+\frac{1-v}{2} \gamma_{x y}^{2}\right] d x d y \\
& V_{2}=\int_{-b-a}^{a} \int_{w} \cdot d x d y \\
& V=V_{1}-V_{2} \\
& \frac{\partial V}{\partial u_{0}}=\frac{\partial V}{\partial v_{0}}=0 \\
& \Rightarrow u=f_{1}\left(w_{0}, \varepsilon_{i n}, x, y\right) \\
& \Rightarrow v=f_{2}\left(w_{0}, \varepsilon_{i n}, x, y\right) \\
& \Rightarrow w=f_{3}\left(w_{0}, \varepsilon_{i n}, x, y\right) \\
& \frac{\partial V}{\partial w_{0}}=0 \\
& \Rightarrow w_{0}=f\left(p, \varepsilon_{i n}\right)
\end{aligned}
$$

Knowing the maximum deflection $\mathrm{w}_{0}$ of the membrane (at the center of the membrane under various loading pressure) from WYKO profile data and a Poisson ratio of 0.4 for parylene-c [11], Young's modulus and intrinsic stress ( $\mathrm{E}$ and $\sigma_{\text {in }}$ respectively) are obtained by fitting the load-deflection curves with the derived $w_{0}=f\left(p, \varepsilon_{\mathrm{in}}\right)$ as shown in Fig.6.

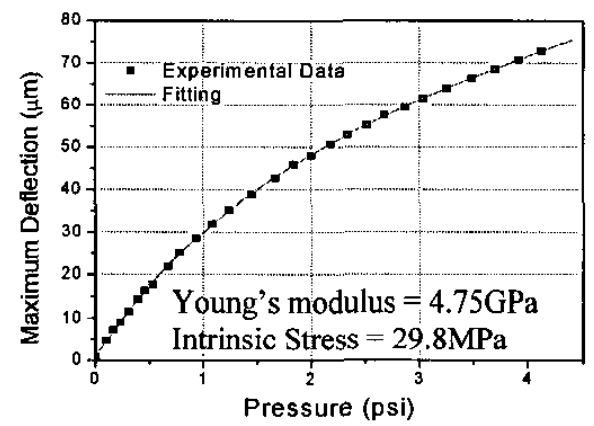

Fig.6 Parameters fitting with 2-step displacement model.

It is found that parameters fitted from our model is very agreeable with those from Tabata's model[10] as 
shown in Table I. Finally, the stress distribution over the whole membrane given a known $\mathrm{w}_{0}$ can be calculated

\begin{tabular}{|c|c|c|}
\hline & Tabata's work & $\begin{array}{c}\text { This } \\
\text { model }\end{array}$ \\
\hline $\begin{array}{c}\text { Young's Modulus } \\
(\mathrm{GPa})\end{array}$ & 4.77 & 4.75 \\
\hline $\begin{array}{c}\text { Intrinsic Stress } \\
(\mathrm{MPa})\end{array}$ & 33.8 & 29.8 \\
\hline
\end{tabular}

Table I. Model comparisons.

with the generalized Hook's law (Eq.2)[12]. The calculation predicts that the maximum stress will occur in the long edge middle of the membrane. This prediction is confirmed by the plastic deformation picture shown in Fig.4. Knowing the onset pressure of plastic deformation to be 4psi we can then determine the corresponding yield strength to be $59 \mathrm{Mpa}$ (Fig.7), which is first reached at the middle points of two long membrane sides.

$$
\begin{aligned}
& \sigma_{x}=\frac{E}{1-v^{2}}\left(\varepsilon_{x}+v \varepsilon_{y}\right) \\
& \sigma_{y}=\frac{E}{1-v^{2}}\left(v \varepsilon_{x}+\varepsilon_{y}\right)
\end{aligned}
$$

Eq.2 Generalized Hook's law. $\sigma_{\mathrm{x}}, \sigma_{\mathrm{y}}$ are stresses in the $\mathrm{x}$ and $\mathrm{y}$ directions respectively.

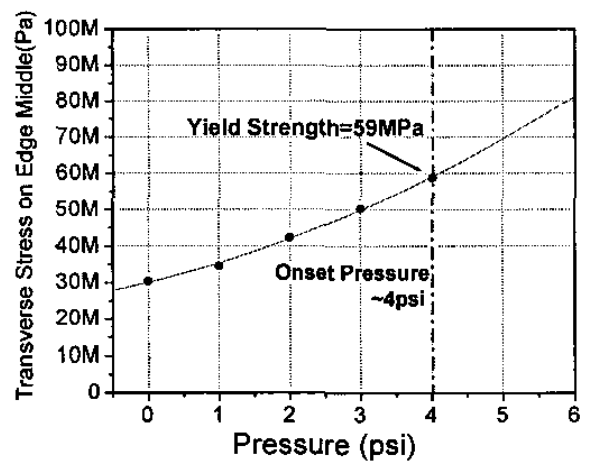

Fig.7 Yield strength derived from energy model and load-deflection experiment.

\section{B. Balloon Model}

To further verify our model, the balloon model[3], Fig.8, is used to analyze the stress distribution at the center of parylene membrane on a new chip. Under the assumption that bending energy is negligible compared with membrane stretching energy (especially true around the membrane center point), balloon model can be applied in our case to calculate stress value at the membrane center. To do so,

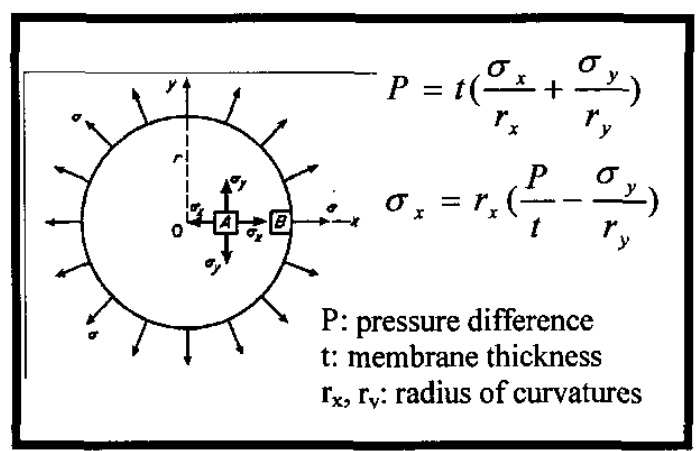

Fig.8 Balloon model for stress analysis.

WYKO surface profiler is used to take the profile pictures of membranes under uniform pressure loading. Average profiles under fixed loading pressure are taken from multiple profile measurements to reduce high frequency profile noise which is probably contributed by optical effect or environmental vibration. The radius of curvatures at the membrane center can then be extracted from the average profile data (Fig.9) using Eq.3. Under

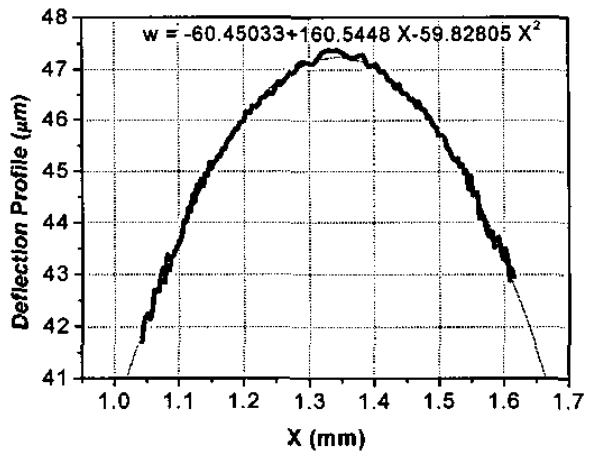

Fig.9 Average WYKO profile data across the membrane center point fitted with second order polynomial curve.

$$
\begin{aligned}
& \frac{1}{r_{x}}=\left\{1+\left(\frac{d w}{d x}\right)^{2}\right\}^{-3 / 2} \frac{d^{2} w}{d x^{2}} \\
& \frac{1}{r_{y}}=\left\{1+\left(\frac{d w}{d y}\right)^{2}\right\}^{-3 / 2} \frac{d^{2} w}{d y^{2}}
\end{aligned}
$$

Eq.3 Formulae for extracting radius of curvatures at the membrane center from average WYKO profile data. 
the assumption that $\sigma_{y}=\sigma_{\text {in }}$ in the membrane center, stress value $\sigma_{\mathrm{x}}$ at the membrane center is then calculated and compare with the value from the 2-step displacement model as in Fig.10. Good agreement is again observed.

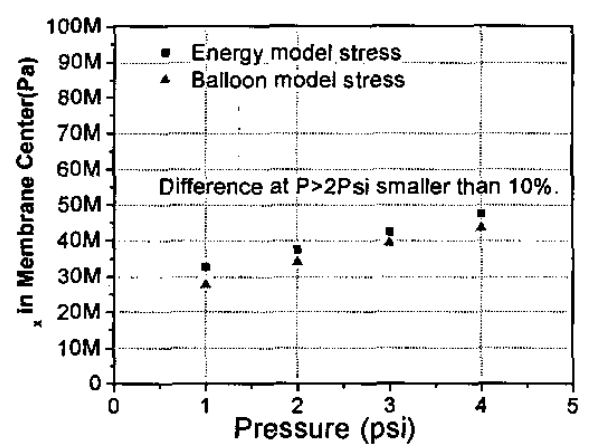

Fig.10 Energy model v.s balloon model.

\section{CONCLUSIONS}

In conclusion, a 2-step displacement approach with energy minimization method is developed to include both contributions from large intrinsic stress and large deflection effects to the total stress distribution across the whole membrane under a uniform pressure loading. The proposed model is used with the membrane loaddeflection experiment to determine the yield strength of thin-film parylene-c to be $59 \mathrm{Mpa}$. The proposed model is verified by comparing analysis results with other known approaches. It is an analytic model suitable for the calculation of stress distribution on the membrane for yield strength measurement or related purposes.

\section{ACKNOWLEDGMENTS}

This work is supported by the NSF Center for Neuromorphic Systems Engineering (CNSE) at Caltech.

\section{REFERENCES}

[1] Timoshenko, "Theory of Plates and Shells", $2^{\text {nd }}$, 1959.

[2] Parylene-C coating services, "Properties of Parylene", http://www.parylene.com.

[3] Timoshenko, et al., "Elements of Strength of Materials", $2^{\text {nd }}, 1940$.

[4] X. Q. Wang, Q. Lin, and Y. -C. Tai, "A Parylene Micro Check Valve," Proceedings of the $12^{\text {th }}$ IEEE International Conference on Micro Electro Mechanical Systems, (MEMS '99) Florida, U.S.A, Jan. 17-21, 1999, pp. 177-182.

[5] L. Licklider, et al., "A micromachined chip-based electrospray source for mass spectrometry," Analytical Chemistry, vol. 72, pp. 367-375, 2000.

[6] J. R. Webster, et al., "An Inexpensive Plastic Technology for Microfabricated Capillary Electrophoresis Chips," Micro Total Analysis Systems '98, Banff, Canada, 1998.

[7] C. Grojean, X. Yang and Y.C. Tai , "A

Thermopneumatic Peristaltic Micropump",

Transducers'99, pp 1776-1779.

[8] W. Sim, et al., "Fabrication, Test and Simulation of a Parylene Diaphragm," Transducers 2001, Munich, Germany, 2001.

[9] D. M. Schneider, J. Maibach and Ernst Obermeier, Journal of Micromechanical Systems. vol. 4, no. 4, pp. 238-241, 1995.

[10] Osamu Tabata, Ken Kawahata, Susumu Sugiyama and Isemi Igrashi, "Mechanical Property Measurements of Thin-Films Using Load Deflection of Composite Rectangular Membranes," Sensors and Actuators, vol. 20, pp. 135-141, 1989.

[11] Theodore A. Harder, Tze-Jung Yao, Qing He, ChiYuan Shih and Yu-Chong Tai, "Residual Stress in Parylene C" Proceedings of the $15^{\text {th }}$ IEEE International Conference on Micro Electro Mechanical Systems (MEMS '02) pp. 435-438.

[12] Egor P. Popov, "Engineering Mechanics of Solids", Prentice Hall, 1990. 